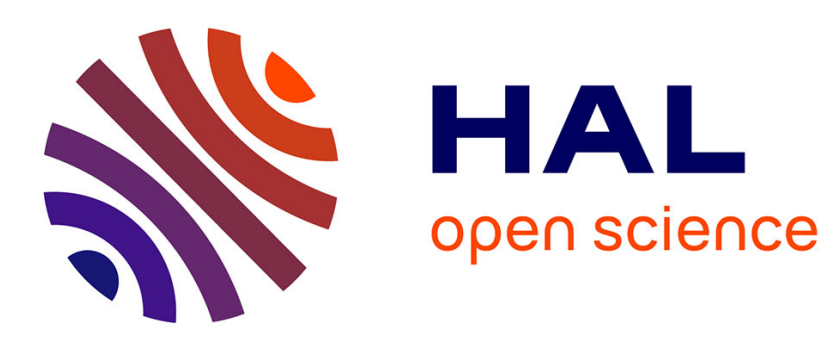

\title{
Le nombre et la part des immigrés dans la population: comparaisons internationales
}

Gilles Pison

\section{To cite this version:}

Gilles Pison. Le nombre et la part des immigrés dans la population: comparaisons internationales. Population et sociétés, 2019, 563, pp.4. hal-02081741

\section{HAL Id: hal-02081741 https://hal.science/hal-02081741}

Submitted on 27 Mar 2019

HAL is a multi-disciplinary open access archive for the deposit and dissemination of scientific research documents, whether they are published or not. The documents may come from teaching and research institutions in France or abroad, or from public or private research centers.
L'archive ouverte pluridisciplinaire HAL, est destinée au dépôt et à la diffusion de documents scientifiques de niveau recherche, publiés ou non, émanant des établissements d'enseignement et de recherche français ou étrangers, des laboratoires publics ou privés. 


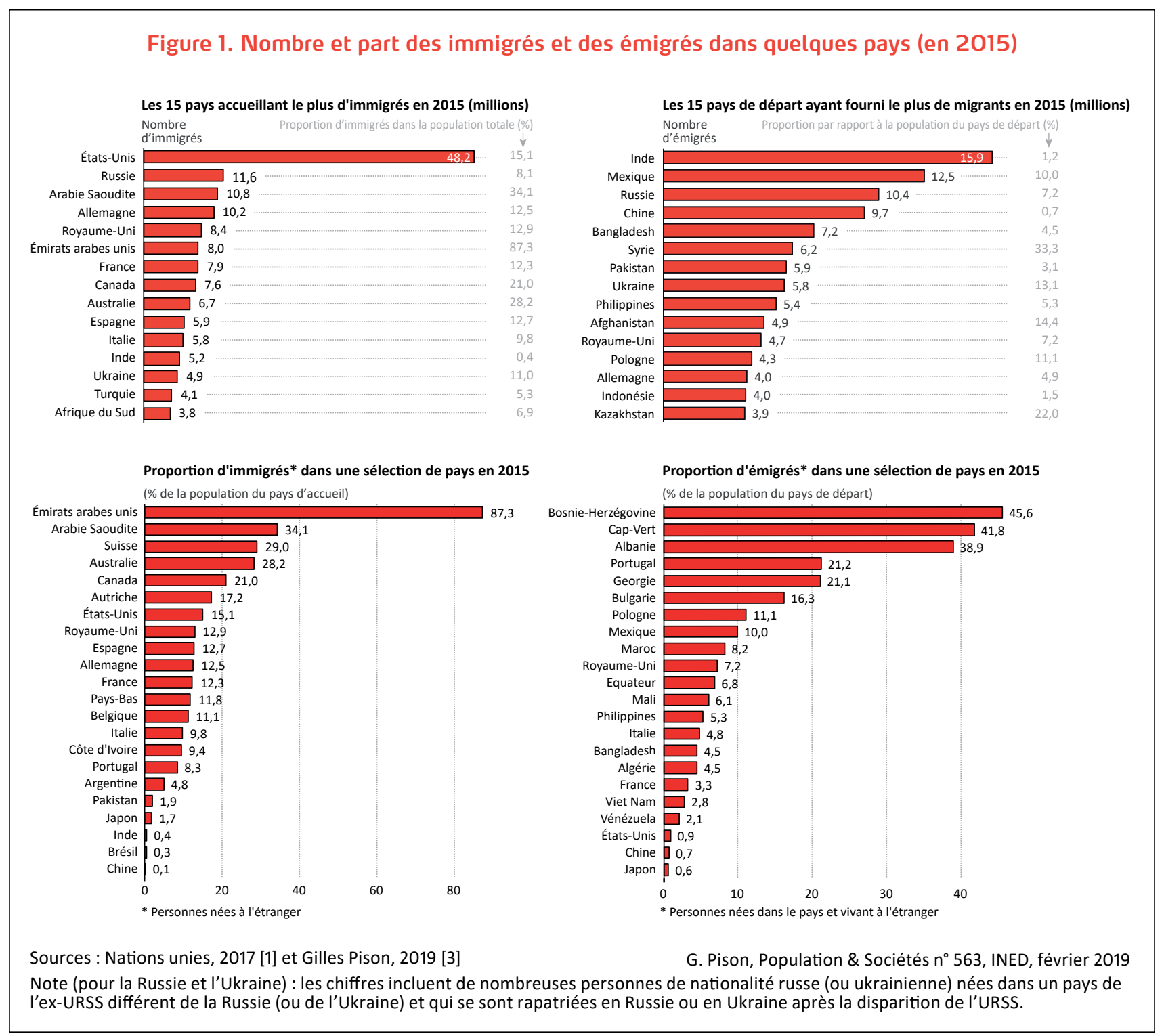

coup plus élevé. Les migrations de province à province, qui ont pris beaucoup d'importance ces dernières années, seraient en effet alors comptées comme des migrations internationales et non plus comme des migrations internes (voir définitions en encadré). En sens inverse, si l'Union européenne formait un seul pays, la part des immigrés diminuerait sensiblement, puisque les ressortissants d'un autre pays de l'Union n'en feraient plus partie. L'importance relative des deux types de migration, interne et internationale, est donc fortement liée au découpage du territoire en nations.

\section{Les États-Unis et la France, pays d'immigration ancienne}

La proportion d'immigrés dans un pays reflète l'importance des flux d'immigration passés. Ainsi, les ÉtatsUnis, avec $15 \%$ de la population née à l'étranger en 2015 (48 millions de personnes), est le premier pays d'accueil des migrants, malgré l'imposition des quotas par nation, qui avaient fortement ralenti les flux de 1924 à 1965. Il le reste aujourd'hui avec un solde migratoire (différence entre les entrées et les sorties de personnes de son territoire) estimé à un peu plus d'un million de personnes en 2015 [1].

La France est également un vieux pays d'immigration, avec des migrants venus au XIX ${ }^{e}$ siècle des pays voisins-Belgique, Angleterre, Allemagne, Suisse -, puis au $\mathrm{XX}^{\mathrm{e}}$ siècle, après la Première Guerre mondiale, de Pologne, d'Italie, d'Espagne et, après la Seconde Guerre mondiale, du Portugal, du Maghreb, puis plus récemment, d'Afrique subsaharienne et d'Asie. Dans les années 1950 et 1960, le solde migratoire était, proportionnellement à la population, plus élevé en France qu'aux États-Unis (en moyenne près de 4 pour mille habitants par an, contre 2 pour mille), du fait des quotas d'entrée. Depuis 1970 c'est l'inverse, le flux d'entrée s'est réduit en France alors qu'il a plutôt augmenté aux ÉtatsUnis, notamment dans les années 1990. Le solde migratoire se situe autour de 3 pour mille par an en moyenne aux États-Unis depuis 2000, contre deux fois moins (près de 1,5 pour mille) en France. Mis à part ces différences conjonc- 
turelles, les deux pays ont en commun une longue histoire d'immigration, avec un flux d'entrée qui, même modeste pendant certaines périodes, s'est maintenu de façon quasi ininterrompue sur plus d'un siècle.

Dans ces pays, la population immigrée s'est constituée progressivement. Les migrants, qu'ils soient venus pour travailler ou rejoindre un membre de leur famille déjà sur place, ont été nombreux ensuite à rester dans le pays d'accueil. Ils y ont fondé une famille, y ont eu des enfants, y ont vieilli. Une partie des immigrés sont retournés dans leur pays de naissance ou sont allés dans un autre pays d'accueil, alors que d'autres sont décédés sur place. La population des immigrés résidant actuellement dans le pays d'accueil résulte du cumul des vagues d'immigration successives, diminué des pertes subies par chacune en raison des départs et des décès.

\section{L'Espagne, nouveau pays d'immigration}

En Espagne, la proportion d'immigrés (13 pour mille en 2015) est du même ordre qu'aux États-Unis ou en France mais, à la différence de ces derniers pays, la population immigrée s'est constituée sur un temps très court. L'Espagne était un pays d'émigration jusqu'à la fin des années 1980. Ce n’est que depuis le début des années 1990 qu'elle est devenue un pays d'immigration. Le flux d'entrée d'immigrés s'est progressivement accru jusqu'à un niveau très élevé, le solde migratoire positif dépassant 600000 personnes par an entre 2002 et 2007, soit, rapporté à la population (43 millions en 2005), un taux de 15 pour mille, près de dix fois plus élevé qu'en France à la même époque. La crise financière de 2008 a fortement réduit le flux d'entrée de migrants et une partie des immigrés est repartie, ce qui a légèrement réduit la proportion d'immigrés qui reste toutefois importante. La courte durée de la période d'immigration a été compensée par un apport migratoire massif, la proportion d'immigrés correspondant finalement à celle de la France. Mais la population immigrée est différente dans les deux pays : l'installation des immigrés vivant en France est en moyenne plus ancienne que celles des immigrés vivant en Espagne. De ce fait, ils sont plus âgés.

\section{Le nombre et la part des émigrés : difficiles à mesurer}

Tout immigré est aussi un émigré pour le pays qui l’a vu naître. Même s'il s'agit des mêmes personnes à l'échelle mondiale, quand on s'intéresse à un pays particulier et que l'on souhaite en connaître la population des émigrés, les informations disponibles sont souvent moins bonnes que pour les immigrés. Les pays sont sans doute moins soucieux de dénombrer leurs émigrés que leurs immigrés, les premiers n'étant plus résidents, et n'occasionnant plus de dépenses publiques sous forme d'équipements et d'infrastructures, contrairement aux seconds. Mais les émigrés contribuent souvent de façon importante à l'économie de

\section{Encadré. Définitions et sources d'information}

Migrant international. Les Nations unies recommandent de retenir comme migrant international toute personne changeant de pays de résidence habituelle pour une durée de séjour d'au moins un an, quel qu'en soit le motif. Le franchissement d'une frontière internationale, avec changement de résidence habituelle, différencie la migration internationale de la migration interne qui s'effectue à l'intérieur des frontières d'un État.

Étranger. Personne qui ne possède pas la nationalité du pays où elle réside.

Immigré. Personne née dans un autre pays que celui où elle réside, qui a donc franchi une frontière (ou plusieurs) depuis sa naissance. Elle peut avoir la nationalité de son pays de naissance ou avoir une autre nationalité, notamment celle du pays dans lequel elle réside. Dans le premier cas, elle est étrangère, et dans le dernier, elle ne l'est pas, ayant la nationalité du pays où elle habite. Il est convenu de réserver la dénomination d'immigré aux seules personnes " nées étrangères à l'étranger ", en excluant les personnes nées à l'étranger de parents expatriés, ayant donc la nationalité de leur pays de résidence depuis leur naissance.

Solde migratoire. Différence entre le nombre de personnes entrées dans le pays au cours d'une période donnée et le nombre de celles qui en sont sorties.

\section{Les sources d'information}

Le nombre d'immigrés est habituellement estimé dans chaque pays grâce au recensement, qui demande à chaque personne résidente son lieu et pays de naissance.

Les flux annuels d'immigration sont estimés à l'aide de sources administratives - délivrance des permis de séjour ou de travail, registre de population. Concernant les flux d'émigration, peu de pays disposent de système permettant d'observer les départs de migrants de leur territoire. Les statistiques migratoires sont donc produites à partir de l'observation des arrivées dans les pays d'accueil.

Les Nations unies, comme d'autres organisations, s'efforcent de rassembler l'ensemble des données démographiques publiées par les offices nationaux de statistique et, en les corrigeant éventuellement, élaborent leurs propres statistiques [1].

leur pays de départ par l'envoi d'argent et, dans certains cas, ils peuvent toujours voter, ce qui justifie de mieux connaître leur population. La moins bonne connaissance des émigrés tient également aux sources statistiques. Les arrivées de migrants sont mieux enregistrées que les départs. Et le nombre d'émigrés est souvent estimé à partir des statistiques sur les immigrés dans les différents pays d'accueil (voir encadré).

Le nombre d'émigrés varie beaucoup d'un pays à l'autre. L'Inde se trouve en tête en 2015 avec près de 16 millions de personnes nées dans ce pays et vivant dans un autre pays (figure 1). Le Mexique occupe la seconde place avec plus de 12 millions d'émigrés vivant principalement aux ÉtatsUnis. En termes de proportion, la Bosnie-Herzégovine 
détient un record: on compte un Bosnien vivant à l'étranger pour deux vivant au pays, ce qui signifie que le tiers des personnes nées en Bosnie-Herzégovine ont émigré. L'Albanie est dans une situation proche, ainsi que le CapVert, pays insulaire dénué de ressources.

Certains pays sont à la fois des terres d'immigration et d'émigration. C'est le cas par exemple du Royaume-Uni, qui compte 8,4 millions d'immigrés et 4,7 millions d'émigrés en 2015. Les États-Unis comptent un nombre appréciable d'expatriés (2,9 millions en 2015), mais comparativement aux immigrés (48 millions à la même date), c'est dix-sept fois moins. La France est dans une situation intermédiaire : d'après les décomptes dans les recensements du monde entier, elle compterait 2,9 millions d'expatriés en 2015, soit autant que les États-Unis, mais $40 \%$ de moins que le Royaume-Uni ; ses émigrés seraient quatre fois moins nombreux que ses immigrés [1]. Enfin, certains pays paraissent relativement fermés jusqu'ici aux migrations, et dans les deux sens. C'est le cas par exemple du Japon, qui compte à la fois peu d'immigrés (seulement $1,7 \%$ de la population en 2015$)$ et peu d'émigrés $(0,6 \%)$.

\section{Les immigrés : moins de $\mathbf{4} \%$ de la population mondiale}

Les immigrés seraient au total 258 millions en 2017 d'après les Nations unies [1]. Ils ne représentent qu'une faible minorité de la population mondiale $(3,4 \%)$, la plupart des humains vivant dans leur pays de naissance. La proportion d'immigrés n'a que très légèrement augmenté au cours des dernières décennies (elle était de $2,9 \%$ il y a 30 ans, en 1990 , et de $2,3 \%$ il y a 55 ans, en 1965). Elle a sans doute également peu changé en 100 ans.

En revanche, la répartition des immigrés n'est pas la même qu'il y a un siècle. L'un des changements survenus depuis est le « renversement des flux migratoires », entre le Nord et le Sud, selon l'expression d'Alfred Sauvy, les pays du Sud fournissant désormais une part importante des migrants internationaux. Ceux-ci se répartissent aujourd'hui en trois groupes d'importance numérique à peu près égale (figure 2) : les migrants nés au Sud et vivant au Nord (89 millions en 2017 d'après les Nations unies [1]), les migrants Sud-Sud (97 millions), qui ont migré d'un pays du Sud vers un autre pays du Sud, et les migrants NordNord (57 millions). Le quatrième groupe des personnes nées au Nord et ayant migré au Sud, qui dominait il y a un siècle, est nettement moins important numériquement (14 millions). Le tableau mondial des migrations interna-

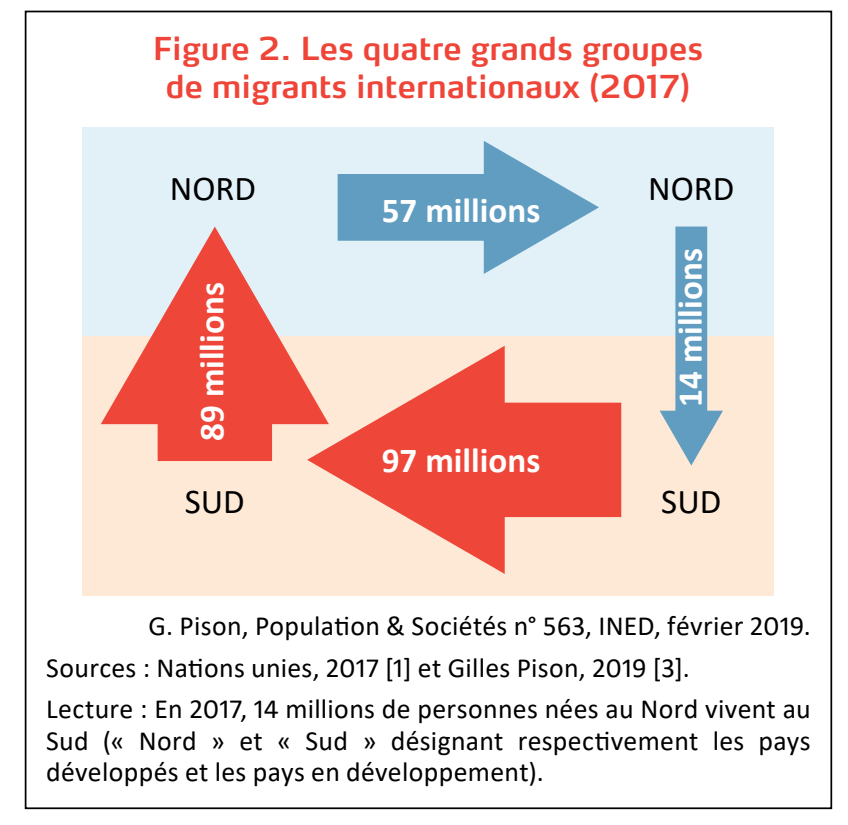

tionales n'aura finalement guère été modifié par les flux de migrants générés depuis 2015 par les conflits au MoyenOrient, malgré leur importance, notamment en Europe.

\section{Références}

[1] Nations unies, Trends in international migrant stock: the 2017 Revision, et International Migration Report 2017, https://www. un.org/en/development/desa/population/migration/data/index. shtml

[2] Gildas Simon, « Les migrations internationales », Population et Sociétés, $n^{\circ} 382$, septembre 2002, 4 p., https://www.ined.fr/ fichier/s_rubrique/18801/pop_et_soc_francais_382.fr.pdf

[3] Gilles Pison, Atlas de la population mondiale, Éditions Autrement, 2019, $95 \mathrm{p}$.

Résumé
Les États-Unis sont le pays du monde ayant sur son sol le
plus grand nombre d'immigrés (personnes nées à
l'étranger) : 48 millions. C'est près de cinq fois plus que
l'Arabie saoudite ( 11 millions) et six fois plus que le Canada
(7,6 millions). Mais proportionnellement à leur taille, ces
deux derniers pays ont nettement plus d'immigrés : $34 \%$
et $21 \%$, contre $15 \%$ aux États-Unis.
Immigrés, émigrés, migrations internationales, comparaisons
internationales, monde.

\footnotetext{
Ined : 133, boulevard Davout, 75980 Paris, Cedex 20 Directrice de la publication : Magda Tomasini Rédacteur en chef : Gilles Pison Éditrice : Marie-Paule Reydet Graphiste : Isabelle Milan Impression : Mérico Delta Print, Bozouls, France D. L. 1er trim. $2019 \cdot$ ISSN 01847783 Numéro 563 - Février 2019 - Population \& Sociétés
}

Bulletin mensuel d'information de l'Institut national d'études démographiques

Retrouvez Population et Sociétés dès sa parution sur le site internet de l'Ined et abonnez-vous :

wwww.ined.fr/fr/publications/population-et-societes

Contact :

edition@ined.fr

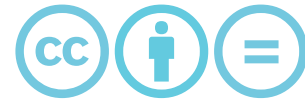

Cet article peut être reproduit sur papier

ou en ligne gratuitement en utilisant notre licence Creative Commons 